\title{
7 Wann ist unser Zusammenleben fair? John Rawls' Beitrag zur modernen Moralphilosophie
}

\author{
7.1 Von Immanuel Kant zu John Rawls \\ 7.2 Rawls' Ethik politischer Gerechtigkeit
}

\section{1 | Von Immanuel Kant zu John Rawls}

Deutscher Idealismus: Kants Philosophie bildet den Auftakt zu philosophischen Systembildungen, die der Rubrik Deutscher Idealismus zugeordnet werden. Ihnen ist der Versuch gemeinsam, in Kants System verbleibende Spannungen und Dualismen zu überwinden. Dies zeigt sich beispielsweise in Johann Gottlieb Fichtes Radikalisierung der kantischen Bewusstseinsphilosophie. Sie erklärt auch noch den von Kant nicht angetasteten Rest eines subjektunabhängigen Seins, das bestimmungslose und an sich unerkennbare Ding an sich, zum Ergebnis einer aktiven Setzung des Erkenntnissubjekts. Es zeigt sich auch in Georg W. F. Hegels Modell dialektischer Entwicklung. Es soll Polaritäten wie die zwischen Begriff und Gegenstand, Subjekt und Objekt oder Moralität und Sittlichkeit zwar nicht eliminieren, aber doch vermitteln und saufheben ihres Harmonisierungsbestrebens sind die Ansätze des Deutschen Idealismus oft von einer tiefen Ambivalenz gekennzeichnet. Sie zeigt sich etwa in Fichtes Naturrechtsbuch (Fichte 1962, Bd. I.3 und I.4). Fichte sucht dort einerseits nachzuweisen, dass Subjekte nur als soziale und leibliche Wesen gedacht werden können. Andererseits will er sogar noch Sozialität und Leiblichkeit aus der schöpferischen Subjektivität des einen Erkenntnissubjekts herleiten. Erkennbar wird die Ambivalenz auch in den radikalen Positionsänderungen in Fichtes und Schellings Denken. Sie zeigt sich schließlich auch in der Offenheit der Hegelschen Philosophie für ganz unterschiedliche, materialistische oder idealistische, Weiterentwicklungen.

Intersubjektivität und Anerkennung: Kants transzendentale Bewusstseinsphilosophie konzipiert Vernunft als Bewusstseinsapparat von Vernunftwesen überhaupt. Die zentrale Rolle der Intersubjektivität, der Sprachlichkeit und der Geschichtlichkeit der menschlichen Vernunft wird weitgehend ausgeblendet. Zwar entwickelt Kant eine aufklärerische Geschichtsphilosophie. Auch zeigt er sich als scharfsinniger Beobachter der Strukturen, Gepflogenheiten und Fallstricke des sozialen Zusammenlebens. Er formuliert sogar explizit eine Kritik des epistemologischen Solipsismus, der es »für unnöthig [hält], sein Urtheil auch am Verstande Anderer zu prüfen; gleich als ob er dieses Probirsteins [...] gar nicht bedürfte« (Anthropologie, AA, Bd.7, S. 128; zum Begriff siehe Kap.2.2). Dessen ungeachtet geht Kant davon aus, dass ein einsames Vernunftwesen grundsätzlich über alle nötigen Erkenntnisbedingungen verfügen könnte,

Ambiguität des Deutschen Idealismus 
Probleme der

kantischen Erkenntnistheorie

Fichte 1962, Bd. I.3, S. 380

Anerkennung als Schlüsselbegriff die nötig sind, um seine Wahrnehmungen strukturieren, und die in ihr anzutreffenden Phänomene verstehen und erklären zu können. Diese Phänomene werden als kausal determiniert erfahren. Dies hat zur Folge, dass die Sphäre der Subjektivität, der Spontaneität und der menschlichen Freiheit auf einen Punkt jenseits der möglichen Erfahrung - das transzendentale Ich - zusammenschrumpft. Damit wird es unmöglich, eine soziale Mitwelt, d.h. eine Sphäre der Begegnung mehrerer Subjekte, des freien Austauschs und der sozialen Interaktion, innerhalb der erfahrbaren Wirklichkeit zu konzipieren. Praktische Freiheit ist nur ıhinter` dieser Wirklichkeit zu denken, kann sich jedoch niemals als solche darin manifestieren. Diese erkenntnistheoretische These führt zu Problemen für die Ethik. Beispielsweise lässt sie die Frage unlösbar erscheinen, wie und mit welchem Recht wir innerhalb der uns erscheinenden Phänomene so etwas wie zurechenbare Handlungen und zurechnungsfähige Akteure identifizieren oder auch nur empirische Indizien für sie finden könnten. Fichte beschreibt dieses Problem in großer Klarheit, indem er fragt:

"[W]ie kommen wir dazu auf einige Gegenstände der Sinnenwelt den Begriff der Vernünftigkeit überzutragen, auf andere nicht; welches ist der charakteristische Unterschied beider Klassen? Kant sagt: handle so, daß die Maxime deines Willens Princip einer allgemeinen Gesezgebung seyn könne. [...] Aber wer soll denn in das Reich, das durch diese Gesezgebung regiert wird, mit gehören, und Antheil an dem Schutze derselben haben? [...] Nun sagt man mir: es versteht sich, daß nur von Wesen, die der Vorstellung von Gesetzen fähig sind, also von vernünftigen Wesen, die Rede sey; und ich habe zwar statt des einen unbestimmten Begriffs einen andern, aber keinesweges eine Antwort auf meine Frage. Denn, wie weiß ich denn, welches bestimmte Objekt ein vernünftiges Wesen sey [...].“

Fichte selbst begnügt sich allerdings seinerseits mit der Antwort, »[d]ie Natur " habe »diese Frage längst entschieden« (ebd.). Das hat philosophische Gründe, denn Fichte legt zwar dar, dass sich niemand als Vernunftwesen verstehen könne, ohne sauch andere endliche Vernunftwesen ausser sich anzunehmen« (ebd., S. 340). Auch Fichte kann jedoch nicht erklären, wie wir in unserer Erfahrung zu diesen Wesen als Mit-Vernunftwesen Zugang erhalten, denn erweitert den kantischen Erfahrungsbegriff, der am Modell der newtonschen Physik orientiert ist, nicht in einer Weise, die einen solchen Zugang zur sozialen Mitwelt ermöglichen würde. Allerdings führt mit dem Begriff interpersonaler Anerkennung einen wichtigen Ansatzpunkt für eine solche Erweiterung des Erfahrungsbegriffs ein. Vermittelt durch Hegel wird dieser Begriff zunehmend zu einem Schlüsselkonzept moral- und sozialphilosophischer Theorien, die in den Strukturen der Intersubjektivität selbst die wesentliche Grundlage sozialer Normierungen erblicken (vgl. ideengeschichtlich Habermas 1988, S. 187241.; Honneth 2018; als Überblick Siep/Ikaheimo/Quante 2018).

Dialogphilosophie und Sorge-Ethik: Auch die ihrerseits an Fichte, den materialistischen Hegel-Schüler Feuerbach und die Sprachphilosophen Wilhelm v. Humboldt und Hamann anknüpfende Tradition der Dialogphilosophie rückt die Rolle der Intersubjektivität in den Mittelpunkt (u. a. Ebner, Buber, Rosenzweig; zur Übersicht und Einordnung Theunis- 
sen 1965). Kritisch gegenüber dem Erfahrungsbegriff Kants stellen sie der objektivierenden Gegenstandserfahrung (bzw. dem »Ich-Es-Verhältnis«) eine dialogische Beziehung (ein »Ich-Du-Verhältnis«) zur Seite (vgl. exemplarisch Buber 1995). Anders als die Ansätze des Deutschen Idealismus zielen sie aber nicht auf die Aufhebung der Polarität, sondern belassen es weitgehend bei der dichotomischen Gegenüberstellung der beiden Weltzugänge (zu den daraus resultierenden Schwierigkeiten vgl. Habbel 1994; Werner 2016). Phänomenologisch orientierte, durch Beiträge der Phänomenologie Husserls und Schelers und der Daseinsanalyse Heideggers modifizierte Einflüsse dieser Tradition sind heute noch in Gestalt einer an Emmanuel Levinas anschließenden `Ethik des Anderen ‘ präsent (Levinas 1987, 1989). Ihrer moralischen Substanz nach weist sie Überschneidungen mit der Sorgeethik (Care Ethics, vgl. Gilligan 1982; Held, 2006; Slote 2007) auf. Ethiken dieses Typs betonen die Ansprüche der konkreten Anderen. Sie sind mehr oder weniger situationistisch angelegt und entsprechend kritisch gegenüber rabstrakten « universalistischen Prinzipienethiken. Die Sorge-Ethik knüpft an vor-kantische Traditionen wie den schottischen Sentimentalismus sowie an die Mitleidsethik an (siehe Kap. 4; Schopenhauer 1988, Bd. 2-4; Schweitzer 1996). Ähnlich dem negativen Utilitarismus (siehe Kap. Alternative Nutzendefinitionen) sieht sie in der Reduzierung des Leidens empfindungsfähiger Wesen den zentralen Gehalt moralischer Verpflichtung. Sie konzipiert diese Verpflichtung aber nicht als Aufgabe einer handlungskonsequentialistischen Optimierung interindividuell aufsummierter Gefühlszustände. Vielmehr betont sie akteursrelative, aus konkreten Beziehungen stammende Verpflichtungen. Während die Sorgeethik heutzutage insbesondere in Kontexten der klinischen Medizinethik und der Pflegeethik vertreten wird, spielt die Mitleidsethik vor allem in der Tierethik eine prominente Rolle (siehe Kap. 11.2).

Sprachpragmatische Wende: Kant vergleicht den Beitrag seiner Transzendentalphilosophie mit dem Übergang zum kopernikanischen Weltbild (kopernikanische Wende). Die vorangehende Philosophie habe versucht, unmittelbaren Zugang zu den Strukturen der objektiven Wirklichkeit zu erhalten, ohne zunächst kritisch die Voraussetzungen der Erkenntnis selbst zu untersuchen. Kants kritischer Idealismus steht für den Wechsel von der Objekt- zur Subjekt-Philosophie. Die konstitutiven Voraussetzungen des Erkennens und Denkens versteht Kant als feste Strukturen des Erkenntnisapparats von Vernunftwesen überhaupt. Eine der entscheidende Leistungen der nach-kantischen Philosophie besteht darin, Kants Frage nach den Voraussetzungen von Erkenntnis und praktischer Deliberation noch weiter zu radikalisieren. Gefragt wird nun auch, inwieweit Erkenntnis und Deliberation ihrerseits von historisch gewachsener sozialer Praktiken abhängig sind; zumal von Strukturen sprachlicher Verständigung. An der Entwicklung dieser neuen Perspektive sind zahlreiche Beiträge aus Semiotik und Pragmatismus, der Logik, Philosophie der Logik, Sprachphilosophie und sprachanalytischen Wissenschaftsund Erkenntnistheorie und der philosophischen Hermeneutik beteiligt. Diese zweite selbstkritische Wendung der philosophischen Perspektive wird, je nach Lesart und Schwerpunktsetzung, als linguistische Wende,

Phänomenologische und sorgeethische Ansätze 
Ethik und Sprachpragmatik

Ethik und Geschichtsphilosophie

Hegel 1970, Bd. 12, S. 32 semiotische Wende, pragmatische oder sprachpragmatische Wende bezeichnet, wobei auch weitere Kombinationsbegriffe (z. B. pragmatischlinguistische Wende) anzutreffen sind (vgl. klassisch Rorty 1967; zur Einordnung und Diskussion Sandbothe 2000).

Diversität sprachpragmatisch geprägter Ansätze: Moralphilosoph/innen ziehen aus den skizzierten Entwicklungen sehr unterschiedliche Konsequenzen, die im Folgenden nur exemplarisch angedeutet werden können. Autor/innen wie Richard Rorty gehen davon aus, dass Pragmatismus und Sprachphilosophie Versuchen einer rationalistischen Moralbegründung in der Tradition Kants den Boden entzogen habe. Diskursethiker/innen und einige andere Vertreter/innen anerkennungstheoretischer und konstruktivistischer (siehe Kap. 8.4.5) Positionen sehen dagegen die Chance einer sprachpragmatisch und hermeneutisch aufgeklärten "Transformation« der Philosophie (Apel 1973), die es ermöglicht, die Kerngehalte der Kantischen Ethik in revidierter Form zu verteidigen. Gerade die Einsicht, dass unsere Vernunft selbst in intersubjektiven Praktiken fundiert ist, soll den Nachweis ermöglichen, dass das Erheben und Einlösen von rationaler Geltungsansprüche (z. B. auf die Wahrheit von Behauptungen oder die Richtigkeit von Handlungen; vgl. Habermas 1981, Bd. 1) immer schon bestimmte intersubjektive Verpflichtungen impliziert. Gezeigt werden soll, dass schon die Rolle einer potentiellen Teilnehmerin an unseren Kommunikations- oder Rechtfertigungspraxen ein grundlegendes »Recht auf Rechtfertigung « (Forst 2005) impliziert (vgl. z. B. Apel 1988; Brune 2010; Darwall 2006).

Historizistische 'Aufhebung' der Moral: Auch die verstärkte Berücksichtigung der Geschichte macht sich in gegenläufigen Tendenzen geltend. Hegel ist überzeugt, dass Kants Moralprinzip allein, ohne Rückgriff auf bereits anerkannte substantielle Normen, keine praktische Orientierung geben kann (`Formalismuseinwand $)$. Kants am Prinzip der individuellen Autonomie orientiertes Verständnis von Moralität möchte er jedoch nicht abstrakt kritisieren, sondern vielmehr in einer Entwicklungsgeschichte der Sittlichkeit raufheben (soll heißen: zugleich bewahren, auf eine höhere Stufe heben und überwinden). Den Begriff der Sittlichkeit verwendet Hegel dabei nicht mehr als gleichbedeutend mit dem der Moralität. Vielmehr versteht er darunter die objektive Seite sozial etablierter Verhaltensnormen. Im Sinne des von Hegel eingeführten Konzepts des objektiven Geistes schlagen sich in den normativen Gehalten der Sittlichkeit freilich selbst die Ergebnisse vernünftiger Reflexion und Kritik nieder. Daher soll die in sozialen Institutionen und Traditionen verkörperte Sittlichkeit auch die von Kant geltend gemachten Ansprüche individueller Autonomie in sich aufnehmen. Als konkrete Sittlichkeit soll sie dadurch selbst zum Medium der Freiheit werden. Tatsächlich interpretiert Hegel die Geschichte der Menschheit idealistisch als einen einer inneren Logik folgenden Emanzipationsprozess des Geistes:

"Die Weltgeschichte ist der Fortschritt im Bewußtsein der Freiheit - ein Fortschritt, den wir in seiner Notwendigkeit zu erkennen haben."

Der Marxismus hält die Vorstellung einer emanzipatorischen Entwick- 
lungslogik der Geschichte aufrecht, nachdem Feuerbach und Marx Hegels idealistische Philosophie materialistisch umgedeutet haben. Da Marx die Triebfeder der historischen Entwicklung nicht mehr in geistigen Widersprüchen, sondern in widerstreitenden ökonomischen Klasseninteressen sieht, erscheinen die Entwicklungen von Moral und Recht jedoch als eher sekundäre Phänomene des ideologischen `Überbaus`. Auch die von der sbürgerlichen` Moral erhobenen universalistischen Geltungsansprüche werden daher geschichtsphilosophisch und ideologiekritisch relativiert. Auch innerhalb der marxistischen Diskussion kommt jedoch wiederholt die Frage auf, inwieweit nicht doch auch der Marxismus selbst an universellen ethischen Ansprüchen orientiert ist (vgl. exemplarisch Sandkühler/De la Vega 1970; andererseits Lukács 1970, S. 110 ff.).

Historismus und Historizismus: Hegel und der Marxismus vertreten die Auffassung, dass die Geschichte einer erkennbaren inneren Gesetzmäßigkeit folgt. Karl R. Popper hat diese Auffassung mit dem Begriff des Historizismus belegt (Popper 2003; der Begriff wird allerdings nicht in einheitlicher Weise gebraucht). Eine noch stärkere Relativierung universalistischer Moralprinzipien als im marxistischen Historizismus findet dort statt, wo die wesentliche Bedeutung soziokultureller Phänomene (einschließlich der Moral und der Ethik) zwar ebenfalls als historisch konstituiert verstanden wird, zugleich jedoch auch noch die Idee einer inneren Entwicklungslogik der Geschichte preisgegeben wird. Entsprechende Auffassungen wurden wiederum schon früh und häufig in polemischer Absicht als historistisch bezeichnet (zum Begriff Scholtz 1971). Eine historistische Auffassung der Moral bedeutet für die normative Ethik, dass die Begründung oder Kritik moralischer Überzeugungen bestenfalls relative, auf einen bestimmten historischen Kontext bezogene Gültigkeit beanspruchen kann. Inwieweit sich ein entsprechender ethischer Relativismus widerspruchsfrei formulieren und verteidigen lässt, wird im nachfolgenden Kapitel diskutiert (siehe Kap. 8.4.2).

Schopenhauers Nihilismus: Kant will die Objektivitätsansprüche wissenschaftlicher Erkenntnis und praktischer Orientierung rechtfertigen. Er will sie gegen die Verwirrungen schützen, die entstehen, sobald wir versuchen, die der Vernunft gesteckten Grenzen zu überschreiten. Seine Auffassung, wonach shinter der für uns zugänglichen, durch unsere Wahrnehmungsformen und Verstandeskategorien geformten Erscheinungswelt ein an sich unerkennbares "Ding an sich" anzunehmen ist, versteht Kant keineswegs als Ausdruck eines Skeptizismus, der die Möglichkeit `echter Erkenntnis in Frage stellt. Just so, als radikaler Skeptizismus, wird Kants Philosophie jedoch von manchen Nachfahren interpretiert. Heinrich von Kleist gerät in Verzweiflung, weil er in Kants Philosophie den Nachweis sieht, dass wir die Wirklichkeit nur wie durch eine verfälschende Brille wahrnehmen können. Auch Hegel findet in Kants Philosophie einen problematischen und sogar selbstwidersprüchlichen Skeptizismus, dem er die Auffassung entgegensetzt, dass Vernunft sich die Überwindung des Gegensatzes von Erscheinung und wahrem Wesen zutrauen kann und muss. Was Kleist in Verzweiflung stürzt und Hegel überwinden will - die Vermutung, dass unser Erkenntnisvermögen uns nur ein Zerrbild der Wirklichkeit darbietet - hält Schopenhauer gerade für Kants wesentliche 
Schopenhauers Mitleidsethik

Nietzsche, KSA Bd. 1, S. $880 \mathrm{f}$.
Einsicht. Er gibt der Skeptizismus-Interpretation der Kantischen Philosophie eine ganz andere, äußerst düstere Lesart: Unsere Vorstellung der Wirklichkeit ist tatsächlich Trug und Schein. Was hinter ihr liegt, ist der Wille - ein a-rationales Streben, das grundsätzlich unerfüllt bleiben muss, weil es außer ihm gar nicht gibt, was ihn befriedigen könnte, und der daher grundsätzlich Leiden bedeutet. Der Wille, der in unseren leiblichen Strebungen und Trieben vorbegrifflich erfahrbar wird, ist das »Ding an sich". Tatsächlich ist er in allen Wesen derselbe, nur die trügerischen Erkenntnisformen unserer Vernunft gaukeln uns vor, dass es verschiedene, mit >Einzelwillen` versehene Individuen gäbe. Dabei steht unsere Vernunft selbst im Dienste individueller Selbstbehauptung. Ist die Trennung von Ich und Du nur eine scheinbare, so ist auch das Leiden aller anderen Wesen im Grunde unser eigenes Leiden. Schopenhauer leitet daraus seine Mitleidsethik ab. Eine vollständige Aufhebung des Leidens ist freilich nur bei vollständiger Aufhebung des Willens möglich. Der Weise, der seinen eigenen Willen überwindet, hebt damit auch die Welt als solche auf. Schopenhauer schreckt vor dieser Konsequenz nicht zurück. Seine Philosophie ist im wörtlichen Sinne nihilistisch. Durch die Lehren des Buddhismus sieht er sich in seiner Auffassung bestätigt.

Vernunftskepsis und Bedeutungsrelativismus: Nietzsche verbindet die von Schopenhauer übernommene sontologischer Relativierung der Ansprüche unseres Erkenntnisvermögens als Ausdruck individueller Selbstbehauptung mit einer sprachphilosophischen und historischen Relativierung unserer Kulturleistungen. In Über Wahrheit und Lüge im außermoralischen Sinne definiert er Wahrheit folgendermaßen:

"Was ist also Wahrheit? Ein bewegliches Heer von Metaphern, Metonymien, Anthropomorphismen kurz eine Summe von menschlichen Relationen, die, poetisch und rhetorisch gesteigert, übertragen, geschmückt wurden, und die nach langem Gebrauche einem Volke fest, canonisch und verbindlich dünken: die Wahrheiten sind Illusionen, von denen man vergessen hat, dass sie welche sind [...]."

Ungeachtet dieses bedeutungstheoretisch begründeten Wahrheitsrelativismus versteht sich Nietzsche selbst als Gegner eines historistischen Wertrelativismus (vgl. KSA, Bd.1, S. 243 ff.), dem alles letztlich gleichgültig ist. Das zeigt sich auch in seiner sprachkritisch unterlegten Moralkritik. In seinem Werk Zur Genealogie der Moral versucht er die historische Entwicklung unserer Moralvorstellungen nachzuzeichnen. Am Bedeutungswandel des Begriffs "gut« meint er zeigen zu können, dass die aristokratische, lebens- und herrschaftsbejahende (griechische) »HerrenMoral « durch eine asketische, lebensverneinende und letztlich nihilistische (jüdisch-christliche) "Sklaven-Moral« ersetzt worden sei (KSA, Bd.V, S. 245 ff.). Die kultur- und ideologiekritische Absicht der begriffs- und kulturgeschichtlichen Skizze ist unverkennbar. Die Darstellung ist durchdrungen von starken, wenn auch nicht immer leicht vereinbaren, Werturteilen. Nietzsche verzichtet jedoch auf den Versuch einer systematischen Darstellung und Begründung dieser Werturteile. Der von ihm vertretene Bedeutungsrelativismus scheint dafür auch kaum Möglichkeiten offen zu lassen. Damit bleibt anscheinend nur die Option, solche Wert- 
urteile dezisionistisch zu interpretieren, das heißt: sie (in der Innenperspektive) als Gegenstand einer nicht mehr hintergehbaren persönlichen >Wertentscheidung « und (in der Außenbetrachtung) als Elemente einer letztlich a-rationalen >Weltanschauung zu verstehen. Diese dezisionistische Subjektivierung der Moral erhält teils auch durch den an Kierkegaard anschließenden Existentialismus Unterstützung (vgl. Kierkegaard 2000). Dezisionismus und Weltanschauungsrelativismus legen es wiederum nahe, die Durchsetzung sozialer Verhaltensnormen ausschließlich als Sache von Sanktion und Machtausübung zu verstehen. Denn der Versuch einer argumentativen Beilegung von Weltanschauungskonflikten über >letzte Wertentscheidungen ist dieser Auffassung zufolge von vornherein zum Scheitern verurteilt.

Ideologische Situation nach 1945: Viele der den öffentlichen Diskurs dominierenden Strömungen des 19. und frühen 20. Jahrhunderts - historistischer und sprachphilosophischer Relativismus, Positivismus, metaethischer Nonkognitivismus, lebensphilosophischer Anti-Rationalismus und existenzialistischer Dezisionismus - stehen dem aufklärerischen Projekt einer rationalen Begründung allgemeinverbindlicher Moralnormen kritisch gegenüber oder stellen dessen Möglichkeit in Frage. Der Neomarxist Georg Lukács (1955) ebenso wie der konservative Rechtsphilosoph Hans Welzel (1990, S. 183) sprechen mit guten Gründen von einer »Zerstörung" bzw. "Selbstzerstörung und Selbstzersetzung" der Vernunft. Übereinstimmend sehen sie darin eine wesentliche Bedingung für die moralische Katastrophe des Nationalsozialismus. An Lukács anknüpfend beschreibt Karl-Otto Apel noch 1973 (Bd. 2, S. 370) die Ideologie der westlichen Nachkriegsgesellschaften als `Komplementaritätssystem`. Ethische Orientierung werde als a-rationale Privatsache, das öffentliche Leben hingegen als tendenziell moralfrei organisierbar (miss-)verstanden. Die Entmoralisierung des öffentlichen Raums erscheint Apel jedoch nicht nur aufgrund der historischen Erfahrungen problematisch, sondern auch im Hinblick auf den durch technolgische Entwicklungen erweiterten Bereich menschlicher Handlungsfolgen (ebd., S. 361). Apel formuliert seine Diagnose in einer historischen Situation, in der neue Partizipationsbestrebungen eine Re-Moralisierung des öffentlichen Raums einfordern. Zugleich und in Zusammenhang damit ist im akademischen Diskurs der Philosophie eine Entwicklung zu verzeichnen, die als Rehabilitierung der praktischen Philosophie bezeichnet wird (Riedel 1972). Motiviert durch den wahrgenommenen Bedarf an politisch-ethischer Orientierung angesichts historischer Erfahrungen, neuer zivilisatorischer Herausforderungen und aktueller politischer Partizipationsbestrebungen, wird sie zugleich durch philosophische Entwicklungen etwa auf dem Feld der Metaethik begünstigt (vgl. Darwall/Gibbard/Railton 1992, S. 121 ff.; siehe Kap. 8). 


\section{2 | Rawls' Ethik politischer Gerechtigkeit}

Rawls' dreifache Bedeutung: Zu den Personen, die am nachhaltigsten zur Rehabilitierung der philosophischen Ethik beigetragen haben, zählt ohne Zweifel John Rawls (Sen 2009, S. 59). Sein Buch Eine Theorie der Gerechtigkeit (A Theory of Justice, Rawls 1999), erstmals 1971 erschienen, prägt die nachfolgende internationale Diskussion in wenigstens dreierlei Weise:

1. Erstens trägt Rawls mit seiner Fokussierung auf Gerechtigkeitsfragen zu einer inhaltlichen Schwerpunktsetzung bei, die bis heute dominierend geblieben ist, auch wenn in den 1980er Jahren Fragen des Guten Lebens wieder verstärkt in den Mittelpunkt treten.

2. Zweitens schlägt er mit dem Konzept des sogenannten Überlegungsgleichgewichts (reflective equilibrium) ein kohärentistisches Modell ethischen Begründens vor, das die Ethik als methodologisch mit anderen Wissenschaften vergleichbar erscheinen lässt und bis heute breite Akzeptanz genießt (s. u.). Dadurch lenkt er zugleich den Blick der angelsächsischen Moralphilosophie von metaethischen Fragen wieder stärker auf Fragen der normativen Ethik (siehe Kap. 8).

3. Drittens trägt er mit seiner Utilitarismuskritik und seinem Plädoyer für ein im weiten Sinne kantisches Moralverständnisses zu einer größeren Akzeptanz deontologischer Ethik bei.

\section{Rawls lesen}

Rawls war in hohem Maße an einer nachvollziehbaren, gründlichen und umsichtigen Darstellung seiner Positionen gelegen. Sein Buch A Theory of Justice ist dadurch einerseits recht umfangreich geraten. Andererseits kommt Rawls durch gezielte Lesehinweise auch noch eiligen Lesern entgegen, die sich nicht in alle Details vertiefen wollen. Die deutsche Übersetzung von 1975 basiert auf einem revidierten Text, der bereits einigen Einwänden Rechnung trägt und auch den späteren englischsprachigen Ausgaben (Rawls 1999) zugrunde liegt. Als erster Zugang zu Rawls' Originaltexten noch besser geeignet ist das erst nach Rawls' Tod herausgegebene und dezidiert als Einleitung verfasste Buch Justice as Fairness: A Restatement (Rawls 2001; dt. 2003). Es hat nicht nur den Vorteil der komprimierteren Darstellung; Rawls reagiert darin auch ausführlicher auf die gegen seine Gerechtigkeitstheorie erhobenen Einwände und rekapituliert die Weiterentwicklungen seiner Position, die er in seinen Beiträgen zum Politischen Liberalismus (Rawls 1993a; dt. 1998) vorgenommen hat.

\subsection{1 | Politische Gerechtigkeitsethik}

Eingeschränkte Zielsetzung: Rawls verfolgt nicht das Ziel, eine umfassende Ethik zu entwickeln, die in allen Fragen des persönlichen Verhaltens Orientierung bieten würde. Vielmehr will er ausschließlich die Frage beantworten, gemäß welchen Prinzipien die Grundstruktur der Gesell- 
schaft (basic structure of society) eingerichtet sein müsste, um als gerecht gelten zu können. Darin liegt eine doppelte Einschränkung: Erstens hinsichtlich des Gegenstandes der ethischen Beurteilung (Grundstruktur) und zweitens hinsichtlich des ethischen Kriteriums (Gerechtigkeit), an dem die Grundstruktur gemessen werden soll.

Grundstruktur als Gegenstand: Unter der Grundstruktur der Gesellschaft versteht Rawls die Konstellation wesentlicher gesellschaftlicher Institutionen, welche die politische Verfassung, die ökonomische und soziale Ordnung bestimmen (Rawls 1999, S. 6 f.). Dazu gehören etwa die Familienstruktur, die Rechtsordnung und die basalen Institutionen politischer Selbstbestimmung, der Regulierung ökonomischer Tätigkeit und der sozialen Vorsorge. Zusammengenommen legen diese wesentlichen Institutionen die Rechte und Pflichten der in ihrer Reichweite befindlichen Menschen fest. Sie bestimmen auch die Verteilung der Gewinne, die aus der sozialen Kooperation hervorgehen. Kurz: Die Grundstruktur bestimmt die Verteilung dessen, was Rawls soziale Grundgüter (social primary goods) nennt (vgl. ebd., S. 54 f.). Diese umfassen sowohl basale Freiheiten als auch soziale und ökonomische Güter, die so beschaffen sind, dass sie von nahezu jeder rationalen Person erstrebt werden. Hinsichtlich dieser sozialen Grundgüter verursacht die gesellschaftliche Grundstruktur häufig tiefgreifende Ungleichheiten. Sie prägen die Lebensaussichten der Betroffenen oft schon von Geburt an. Bereits aus diesem Grund können jene Ungleichheiten nicht - zumindest nicht allein - auf Grundlage individueller Verdienste gerechtfertigt werden. Rawls sucht nun zu klären, inwieweit solche Ungleichheiten überhaupt $\mathrm{zu}$ rechtfertigen sind. Dabei schränkt er den Gegenstand seiner Überlegungen auf die Frage innergesellschaftlicher Gerechtigkeit ein. Individualethische Fragen der Gerechtigkeit im zwischenmenschlichen Umgang klammert er weitgehend aus. Fragen der Gerechtigkeit im Austausch zwischen Staaten behandelt er erst in einem späteren Aufsatz ausführlicher (Rawls 1993b). Ferner legt er seiner Untersuchung die Annahme einer wohlgeordneten Gesellschaft zugrunde, deren Normen von ihren Mitgliedern weitgehend beachtet werden.

Gerechtigkeit als Kriterium: Rawls' Überlegungen konzentrieren sich auf die Frage, wann die Art und Weise, in der die gesellschaftliche Grundstruktur Rechte, Pflichten und Kooperationsgewinne zuteilt, gerecht genannt werden kann. Fragen des guten Lebens stehen nicht im Mittelpunkt der Betrachtung. Dies drückt zwei für Rawls' Moralphilosophie wesentliche Überzeugungen aus. Erstens ist Rawls der Auffassung, dass Gerechtigkeit die »erste Tugend sozialer Institutionen« darstellt. Gerechtigkeit sei für die Legitimität von Institutionen genauso wesentlich wie Wahrheit für die Vertretbarkeit von Theorien:

"Justice is the first virtue of social institutions, as truth is of systems of thought.

Rawls 1999, S. 3 A theory however elegant and economical must be rejected or revised if it is untrue; likewise laws and institutions no matter how efficient and well-arranged must be reformed or abolished if they are unjust. Each person possesses an inviolability founded on justice that even the welfare of society as a whole cannot override. For this reason justice denies that the loss of freedom for some is made right by

Rechtfertigbarkeit sozialer Ungleichheiten 
a greater good shared by others. It does not allow that the sacrifices imposed on a few are outweighed by the larger sum of advantages enjoyed by many."

\section{Zweitens plädiert er für ein deontologisches Verständnis moralischer} Richtigkeit, dem zufolge das Gerechte und Richtige nicht nur eine >Funktion des Guten darstellt; genauer gesagt: dass das Gerechte nicht mit demjenigen identifiziert werden kann, das ein vor-moralisches Gut maximiert (ebd., S.26f.). Nach der von Rawls vertretenen deontologischen Auffassung zielt Gerechtigkeit vielmehr auf den Schutz basaler Rechte aller Moralsubjekte sowie auf die Gewährleistung fairer Chancen auf die Verwirklichung der jeweiligen Lebenspläne. Rawls ist sich allerdings der Tatsache bewusst, dass auch eine solche Konzeption von Gerechtigkeit nicht vollständig auf Annahmen über die Ingredienzien eines guten Lebens verzichten kann. Auch sie muss gewisse Annahmen über die Rationalität von Lebensplänen enthalten und damit auch Urteile über Fragen des guten Lebens fällen. Allerdings soll Rawls' Theorie mit einer vergleichsweise gehaltsarmen bzw. »dünnen" (Rawls 1999, S.347 ff.) Theorie des Guten auskommen. Als Vertreter des Politischen Liberalismus geht Rawls davon aus, dass das gute Leben von Bürgerinnen und Bürgern wesentlich von deren mehr oder weniger unterschiedlichen Projekten eines guten Lebens abhängt und die Aufgabe der Gesellschaft in diesem Zusammenhang vor allem darin besteht, Freiheiten und konkrete Chancen für die Realisierung verschiedener Lebenspläne zu sichern und gerecht zu verteilen.

Das in der politischen Philosophie gebräuchliche Konzept des Politischen Liberalismus, das zur Charakterisierung der rawlsschen Position verwandt wird, ist keineswegs gleichzusetzen mit bestimmten Programmen auf dem Feld der Rechts-, Kultur- oder Wirtschaftspolitik, die ebenfalls - und zwar nach in den USA und Deutschland recht unterschiedlichen Kriterien - als >liberak bezeichnet werden. Soweit sich aus Rawls' Gerechtigkeitstheorie Ansatzpunkte für politische Ziele gewinnen lassen, weisen sie in Richtung eines entwickelten Sozialstaats, der ungleichen Entwicklungen in den Lebensverhältnissen aktiv entgegenwirkt (Rawls 1999, S. xii, Anm. 1 und xiv ff.).

\subsection{2 | Rawls' Überlegungen zur Methode ethischen Begründens}

Kohärentismus und Kontraktualismus: Das Ziel normativ-ethischer Theorien liegt darin, richtige Antworten auf Fragen praktischer Orientierung und ethischer Beurteilung zu geben. Dafür sind sie auf Methoden ethischen Begründens angewiesen. Diese Methoden können wiederum als mehr oder weniger vernünftig - legitim, nachvollziehbar, operationalisierbar, angemessen etc. - beurteilt werden. Sie sind also ihrerseits ein Gegenstand möglicher Begründung oder Kritik. Auch zur Diskussion über 
Begründungsmethoden hat Rawls einen wichtigen Beitrag geleistet. Kennzeichnend für seine Theory of Justice ist zunächst die ausführliche und explizite Auseinandersetzung mit der Methodenfrage. Charakteristisch ferner, dass zwei grundsätzlich voneinander unabhängige methodische Ansätze kombiniert werden, nämlich Kohärentismus und Vertragstheorie. Rawls verbindet die kohärentistische Methode des Überlegungsgleichgewichts (reflective equilibrium) mit dem kontraktualistischen Modell des "Urzustands" (original position). In der an die Theory of Justice anschließenden Diskussion sind beide Methodenbausteine sowie ihr Verhältnis zueinander genauer geklärt worden.

Kohärentismus oder auch Kohärenztheorie der Rechtfertigung nennt man eine Position der Erkenntnistheorie, die besagt, dass die Rechtfertigung von Überzeugungen mit der Bemühung identisch ist, ein möglichst konsistentes und kohärentes Überzeugungssystem zu generieren. Der epistemologische Kohärentismus ist nicht identisch mit einer Kohärenztheorie der Wahrheit, die Wahrheit selbst als eine Form von Kohärenz begreift. Er setzt eine solche Theorie auch nicht zwingend voraus (vgl. genauer Olson 2017).

Eine Entscheidungsprozedur: Bereits 1951 hat Rawls eine Entscheidungsprozedur für die Ethik skizziert. Sie sieht zunächst Kriterien für die Anerkennung von Personen als kompetente moralische Beurteiler/innen (competent moral jugdes) vor. Kompetente moralische Beurteiler/innen benötigten »Tugenden moralischer Einsicht", die mit »intellektuellen Tugenden« vergleichbar seien: Ebenso wie letztere für die Fähigkeit zur Gewinnung kognitiver Einsichten überhaupt wesentlich seien, brauchten wir erstere, um moralische Erkenntnisse gewinnen und faire Urteile bilden zu können (Rawls 1951, S. 181). Zu den Tugenden moralischer Einsicht zählen ein hinreichendes Maß an Intelligenz, hinreichende Kenntnisse über relevante Handlungsumstände, kognitive Offenheit, die Bereitschaft zur Anwendung der Prinzipien induktiven Schließens bei der Meinungsbildung, die Neigung, Gründe für alternative Handlungsoptionen zu prüfen, Selbsterkenntnis und die Bereitschaft zur Selbstkritik. Ferner gehört dazu ein "sympathisierendes Wissen" (sympathetic knowledge) um die jeweiligen Interessen aller möglichen Handlungsbetroffenen (ebd., S. 178 f.). In einem zweiten Schritt werden Kriterien aufgestellt, die festlegen, unter welchen Bedingungen Urteile als wohlerwogene Urteile (considered judgments) zählen können. Dazu gehören die Abwesenheit von Zwang und Befangenheitsgründen, Übersichtlichkeit der Entscheidungsmaterie, subjektive Gewissheit und Beständigkeit des Urteils. Schließlich gehört dazu der Umstand, dass die Urteile »intuitiv« und unmittelbar fallbezogen sind. Die wohlerwogenen Moralurteile sollten also nicht schon selbst das Ergebnis der Anwendung einer ethischen Theorie sein (ebd., S. 181 ff.). In einem dritten Schritt wird eine Explikation der wohlerwogenen Urteile kompetenter moralischer Beurteiler/innen vorgenommen. Auf induktive Weise, also durch den Schluss vom Besonde-

Frühe Überlegungen zur Methode 
Rawls 1951, S. 188

Wechselseitiger Abgleich von Einzelurteilen und Prinzipien ren auf das Allgemeine, wird ein Set allgemeinerer Prinzipien entwickelt. Gemäß dem Prinzip der theoretischen Sparsamkeit sollten diese Prinzipien möglichst einfach gehalten sein. Zugleich sollten aus ihnen möglichst viele der wohlerwogenen Urteile kompetenter Beurteiler/innen begründet werden können (ebd., S. 184 ff.). Zusätzlich sollen diese Prinzipien ihrerseits von kompetenten moralischen Beurteiler/innen als Ergebnis einer offenen und kritischen Diskussion zwanglos akzeptiert werden können:

"[T]he reasonableness of a principle is tested by seeing whether it shows a capacity to become accepted by competent moral judges after they have freely weighed its merits by criticism and open discussion, and after each has thought it over and compared it with his own considered judgments. It is hoped that some principles will exhibit a capacity to win free and willing allegiance and be able to implement a gradual convergence of uncoerced opinion."

Die durch die Explikation wohlerwogener Urteile gewonnenen Prinzipien sollten dann imstande sein, bei der Auflösung moralischer Kontroversen zu helfen. Schließlich sollen sie in einzelne Bereichen auch Korrekturen wohlerwogener Moralurteile ermöglichen: Es soll der Fall sein, dass wir die Prinzipen auch dann noch für vernünftig halten, wenn sie in einzelnen Praxiskontexten wohlerwogenen Einzelurteilen widersprechen (ebd.).

Das Überlegungsgleichgewicht: Rawls’ Methodenskizze lässt eine gewisse Zirkularität erkennen: Wohlerwogene Moralurteile stellen einerseits die Begründungsbasis dar, aus der durch Explikation allgemeinere Moralprinzipien gewonnen werden sollen. Deshalb sollen die wohlerwogenen Urteile selbst intuitiv und sollen sie nicht schon als solche ein Resultat der Anwendung allgemeinerer Prinzipien sein. Andererseits sollen sie aber auf Basis der aus ihnen induktiv gewonnen Prinzipien auch wieder kritisch geprüft und unter Umständen revidiert werden können. Rawls hält diese begrenzte Zirkularität nicht für problematisch. Die Möglichkeit eines wechselseitigen Abgleichs von Prinzipien an Einzelurteilen und Einzelurteilen an Prinzipien (die Ähnlichkeiten mit dem sog. hermeneutischen Zirkel aufweist; vgl. Gadamer 2010) ist vielmehr gerade ein wesentliches Charakteristikum der Methode, auf die er auch in der Theory of Justice baut (Rawls 1999, S.40, Fn. 24). Worauf die Methode abzielt, ist ein sogenanntes Überlegungsgleichgewicht (reflective equilibrium): Einen Zustand größtmöglicher Konsistenz und Kohärenz zwischen unseren vortheoretisch-intuitiven Überzeugungen einerseits und unseren explikativ und induktiv gewonnenen Prinzipien und theoretischen Modellen andererseits (ebd., S. 18, 42 f.).

Ethik und Grammatik: Rawls stellt in der Theory of Justice einen Vergleich zwischen ethischen Theorien und solchen im Bereich der Grammatik an (ebd., S. 41 f., 430). Theorien der Grammatik schlagen Prinzipien vor, die es ermöglichen, die von kompetenten Sprecher/innen intuitiv beherrschte Fähigkeit zur Bildung korrekter Sätze in einer bestimmten Sprache theoretisch nachzukonstruieren. Tatsächlich werden auch Grammatiktheorien einerseits durch die Rekonstruktion intuitiver Sprachver- 
wendungen generiert. Andererseits werden sie aber durchaus auch zur Korrektur einzelner abweichender Sprachverwendungen oder zur Ent-

Moralpraxis und Sprachpraxis scheidung in kontroversen Fällen herangezogen. Auch im Bereich der Grammatik gibt es im Fall hartnäckiger Abweichungen zwischen intuitiven Sprachverwendungen und grammatischen Prinzipien keine Entscheidungsbasis, die von der Sprachpraxis kompetenter Sprecher/innen ganz unabhängig wäre. Vielmehr gilt es, im Hinblick auf intuitive Plausibilität, Konsistenz, Kohärenz und theoretische Sparsamkeit möglichst überzeugende Regeln festzulegen. Falls doch wieder Spannungen auftreten, werden wir in manchen Fällen unsere vor-theoretischen Überzeugungen oder Praktiken, im anderen Fällen hingegen unsere Regeln und unsere theoretischen Annahmen revidieren (zur Diskussion des Grammatikvergleichs und seiner Grenzen vgl. auch Daniels 1996, S. 66 ff.; Gert 1998, S. 4 ff.; Williams 2006, S. 93 ff.).

Einbeziehung nicht-ethischer Theorien: Das von Rawls in der Theorie of Justice angestrebte Überlegungsgleichgewicht soll außer wohlerwogenen Moralurteilen und allgemeinen ethischen Prinzipien auch noch nicht-ethische Überzeugungen einbeziehen. Soweit solche Überzeugungen Implikationen für die jeweilige Plausibilität alternativer moralphilosophischer Positionen haben, spielen auch sie eine Rolle im Rahmen ethischer Rechtfertigungsbemühungen. Beispielsweise ist die identitätstheoretische Vorstellung, dass die Grenzen zwischen verschiedenen Personen von wesentlicher Bedeutung sind, besser mit Moraltheorien kantischen Typs vereinbar, die individuelle Grundrechte vorsehen, als mit dem utilitaristischen Modell einer interindividuellen Nutzenaggregation. Denn da im Rahmen dieses Modells jeder individuelle Nutzen oder Schaden unterschiedlicher Personen so behandelt wird, als ob er einem einzigen Kollektivsubjekt zukäme, trägt es den Identitätsgrenzen zwischen verschiedenen Personen gar nicht unmittelbar Rechnung. Rawls' kohärentistische Begründungsmethode des Überlegungsgleichgewichts geht insofern über die in Rawls' Aufsatz von 1951 skizzierte Entscheidungsmethode hinaus. Denn die Methode des Überlegungsgleichgewichts zielt nicht nur auf Kohärenz zwischen intuitiven Moralurteilen und moralischen Prinzipien, sondern auch noch zwischen diesen beiden und nicht-moralischen Annahmen, beispielsweise Annahmen in Bezug auf das Verständnis personaler Identität (Daniels 1996, S. 21 ff.).

Der Urzustand: Rawls sucht nun zu zeigen, dass es unseren intuitiven Fairnessvorstellungen entspricht, wenn wir die grundlegenden Prinzipien politischer Gerechtigkeit, denen die Grundstruktur der Gesellschaft gerecht werden soll, vom Ergebnis eines bestimmten vertragstheoretischen Gedankenexperiments abhängig machen. Rawls nennt dieses Gedankenexperiment den Urzustand (original position) nennt (Rawls 1999, S. 15 ff., 102 ff.; zur Diskussion Freeman 2016). Die Situation ist erstens dadurch gekennzeichnet, dass die vertragsschließenden Parteien zwar wissen, welche sozialen Rollen und Positionen die Gesellschaft vorsieht, für die sie Entscheidungen treffen, und dass sie einschätzen können, wie sich Entscheidungen über die Grundstruktur auf die Situation verschiedener sozialer Gruppen und auf Individuen mit unterschiedlichen Konzepten des guten Lebens auswirken werden. Sie wissen jedoch nicht, wen sie

Vertragstheoretisches Gedankenexperiment 
Schleier des Nichtwissens

Rawls 1999, S. 11 eigentlich repräsentieren: Welche natürlichen Talente sie besitzen, welche soziale Rolle sie in der Gesellschaft innehaben, welcher Generation sie angehören und welche spezifische Vorstellung des guten Lebens ihnen eigen ist. Die Vertragsparteien befinden sich in dieser Hinsicht, wie Rawls formuliert, hinter einem Schleier des Nichtwissens (veil of ignorance). Ferner sollen die fiktiven Vertragsparteien die alternativen Vorschläge für die Gestaltung der Grundstruktur ausschließlich nach Kriterien klugen Eigeninteresses beurteilen. Insbesondere sollen sie sich weder von Neid noch von Altruismus leiten lassen (Rawls 1999, S.12). Strikte Orientierung am Eigeninteresse und Schleier des Nichtwissens gemeinsam sorgen für die Unparteilichkeit der Entscheidungsprozedur. Die wesentliche Pointe dieses Modells ist dieselbe wie in der Prozedur des fairen Kuchenteilens nach dem Motto »die eine teilt, die andere wählt!«. Da die teilende Person nicht weiß, welches Stück die andere Person wählen wird, wird sie sich, wenn sie lediglich am eigenen Vorteil orientiert ist, bemühen, die Kuchenstücke möglichst gleich attraktiv zu machen:

"Among the essential features of this situation is that no one knows his place in society, his class position or social status, nor does any one know his fortune in the distribution of natural assets and abilities, his intelligence, strength, and the like. I shall even assume that the parties do not know their conceptions of the good or their special psychological propensities. The principles of justice are chosen behind a veil of ignorance. This ensures that no one is advantaged or disadvantaged in the choice of principles by the outcome of natural chance or the contingency of social circumstances. Since all are similarly situated and no one is able to design principles to favor his particular condition, the principles of justice are the result of a fair agreement or bargain. For given the circumstances of the original position, the symmetry of everyone's relations to each other, this initial situation is fair between individuals as moral persons, that is, as rational beings with their own ends and capable, I shall assume, of a sense of justice. The original position is, one might say, the appropriate initial status quo, and thus the fundamental agreements reached in it are fair."

Im Urzustand sind gewissermaßen alle Parteien in der Rolle der Kuchenteilerin, da keine Partei weiß, in welcher Rolle sie jeweils von den Institutionen der Grundstruktur betroffen ist. Der Schleier des Nichtwissens lässt den Parteien daher gar keine andere Wahl als die, die verfügbaren Optionen völlig unparteilich zu beurteilen. Er erzwingt eine Art gedankenexperimentellen Rollentausch. Rawls nennt seine Gerechtigkeitskonzeption Gerechtigkeit als Fairness, weil sie eine faire Prozedur für die Festlegung der Gerechtigkeitsprinzipien vorschlägt, an denen sich die soziale Grundstruktur messen lassen muss. Die Legitimität des Urzustands als Entscheidungsprozedur soll - ganz im Sinne des kohärentistischen Überlegungsgleichgewichts - noch zusätzlich erhöht werden, wenn aus dem Modell Prinzipien resultieren, die ihrerseits von den Bürger/innen als angemessene Explikation ihrer wohlerwogenen vor-theoretischen $\mathrm{Ge}$ rechtigkeitsvorstellungen anerkannt werden.

Das Maximin-Prinzip: Rawls' Urzustand ist lediglich ein Gedankenexperiment. Genauer gesagt beschreibt er die Bedingungen eines Gedan- 
kenexperiments, in dem wir die Prinzipien für eine gerechte Grundstruktur festlegen. Diese Bedingungen sollen erstens fair sein; zweitens sollen sie erlauben, in einer nachvollziehbaren, transparenten und möglichst unkontroversen Weise zwischen alternativen Gerechtigkeitsprinzipien zu entscheiden. Entsprechend macht das Modell des Urzustands Gebrauch von rationalitätstheoretischen Annahmen und entscheidungstheoretischen Verfahren, die auch im Rahmen des Utilitarismus, in ökonomischen Theorien oder im Rahmen konkurrierender Vertragstheorien gebraucht werden. Entsprechend lässt sich der Schleier des Nichtwissens auch in Begriffen der Entscheidungstheorie beschreiben, indem man feststellt, dass die Parteien im Urzustand unter Ungewissheit entscheiden müssen - sie wissen nicht, welche Gruppe sie repräsentieren. Rawls nimmt nun an, dass es für klug ihr Eigeninteresse verfolgende Parteien bei der Entscheidung über die Grundstruktur rational ist, das Maximin-Prinzip zugrunde legen. Das bedeutet, so zu entscheiden, dass die Konsequenzen, die im ungünstigsten Fall eintreten werden, mindestens ebenso gut sind wie die Konsequenzen, die sich bei anderen Entscheidung im dann jeweils ungünstigsten Fall ergeben würden. Warum aber sollte es für die Parteien rational sein, sich am Maximin-Prinzip zu orientieren? Erstens müssen sie damit rechnen, selbst zur am schlechtesten gestellten Gruppe zu gehören, ohne etwas über die Wahrscheinlichkeit, mit der dies der Fall ist, zu wissen. Zweitens wird die Entscheidung über die Grundstruktur der Gesellschaft ihre eigenen Lebenschancen sehr tiefgreifend beeinflussen. Klug am eigenen Interesse orientierte Parteien werden daher dafür Sorge tragen, dass die sozialen Grundgüter so verteilt werden, dass die am schlechtesten gestellten Mitglieder der Gesellschaft in einer mindestens ebenso günstigen Position sind wie es die Mitglieder der am schlechtesten gestellten Gruppe in jeder alternativen Gesellschaftsordnung wären. Sie werden sich also genauso verhalten wie rationale Kuchenteiler/ innen, die damit rechnen müssen, jeweils das kleinste Kuchenstück zu erhalten.

\subsection{3 | Prinzipien politischer Gerechtigkeit}

Zwei Grundsätze der Gerechtigkeit: Rationale Kuchenteiler/innen werden sich bemühen, alle Stücke gleich groß zu schneiden. Rawls nimmt jedoch an, dass das Ergebnis seines Gedankenexperiments nicht einfach die Gleichverteilung aller sozialen Grundgüter wäre. Er hält es vielmehr für plausibel, dass die Parteien sich auf zwei Gerechtigkeitsprinzipien einigen würden, deren erstes die Verteilung von Grundfreiheiten festlegt, während das zweite Prinzip den Zugang zu Ämtern und gesellschaftlichen Optionen sowie die Verteilung sozialer und ökonomischer Ressourcen reguliert. Die Formulierung der in der Theorie der Gerechtigkeit ursprünglich vorgeschlagenen Grundsätze hat Rawls später in einigen Punkten revidiert. In ihrer letzten Fassung lauten die Grundsätze so: 
Rawls 2001, "(a) Each person has the same indefeasible claim to a fully adequate scheme of

S. $42 \mathrm{f}$. equal basic liberties, which scheme is compatible with the same scheme of liberties for all; and

(b) Social and economic inequalities are to satisfy two conditions: first, they are to be attached to offices and options open to all under conditions of fair equality of opportunity; and second, they are to be to the greatest benefit of the least-advantaged members of society (the difference principle)."

Zwischen diesen beiden Prinzipien, deren zweites noch einmal in zwei Teilprinzipien gegliedert ist, gilt eine strikte Hierarchie: Das erste Prinzip (das Prinzip gleicher Grundfreiheiten) soll vor dem zweiten Vorrang haben. Ebenso soll der erste Bestandteil des zweiten Prinzips (der Grundsatz fairer Chancengleichheit) Vorrang vor dessen zweitem Bestandteil haben (dem sog. Differenzprinzip; Rawls 2001, S. 43). Das Differenzprinzip als Gerechtigkeitsgrundsatz ist nicht mit dem entscheidungstheoretischen Maximin-Prinzip zu verwechseln: Am Maximin-Prinzip orientieren sich die Parteien im Urzustand. Das Differenzprinzip ist dagegen eines der von ihnen für die Grundstruktur vorgesehenen Prinzipien gerechter Verteilung. Dass sich die Parteien im Urzustand auf das Differenzprinzip einigen würden, ist freilich eine Folge ihrer Orientierung am Maximin-Prinzip.

Soziale Freiheiten, Ämter, Ressourcen: Die einzelnen GerechtigkeitsgrundGrundgüter sätze betreffen unterschiedliche Arten sozialer Grundgüter: Erstens Grundfreiheiten, zweitens den Zugang zu Ämtern und schließlich drittens ökonomische Ressourcen. Grundfreiheiten müssen gemäß dem ersten Prinzip egalitär zugeteilt werden, also allen gleichermaßen zukommen. Zu diesen Grundfreiheiten zählt Rawls das aktive und passive Wahlrecht, die Rede-, Versammlungs-, Gewissens- und Gedankenfreiheit, die Freiheit der Person und der physischen und psychischen Unversehrtheit, das Recht auf persönliches Eigentum und den Schutz vor willkürlicher Festnahme und Beschlagnahmung gemäß den Prinzipien eines Rechtsstaats (Rawls 1999, S. 53). Das Prinzip der fairen Chancengleichheit (der erste Bestandteil des zweiten Prinzips) ist zwar ebenfalls insofern egalitär, als es allen dasselbe Recht auf faire Konkurrenz einräumt. Es bedeutet freilich nicht, dass alle ein gleiches Anspruchsrecht auf gesellschaftliche Positionen und Ämter haben, sondern nur, dass diejenigen, die dasselbe Maß an Talent, Befähigung und Leistungsbereitschaft haben, in ihrer Konkurrenz um diese Positionen und Ämter dieselben Erfolgschancen haben sollen (ebd., S. 63). Schließlich muss die Verteilung ökonomischer Ressourcen, die aus dem Funktionieren gerechter sozialer Institutionen resultiert, gemäß dem Differenzprinzip die am schlechtesten gestellte Gruppe in eine soziale Lage versetzen, die mindestens genauso gut ist, wie die Lage der jeweils am schlechtesten gestellten Gruppe in jeder anderen denkbaren Gesellschaftsordnung - genauer gesagt: in jeder anderen Gesellschaftsordnung, die zugleich den beiden vorangehenden Gerechtigkeitsprinzipien genügt. Der einschränkende $\mathrm{Zu}$ satz erklärt sich aus der Hierarchie der Gerechtigkeitsgrundsätze. Sie schließt aus, dass Grundfreiheiten oder das Recht auf fairen Wettbewerb gegen ökonomische Vorteile eingetauscht werden. 
Gleichheit und Differenz: Warum aber soll nur ein Teil der Grundgüter egalitär verteilt werden, ein anderer Teil aber nach dem Differenzprinzip? Ein Grund liegt darin, dass die Menge der gesellschaftlich erwirtschafteten Ressourcen keine feststehende Größe ist. Anders als die Menge eines zur Verteilung stehenden Kuchens ist der Umfang der gesellschaftlichen Wertschöpfung variabel. Zudem ist es möglich, dass die von den ökonomischen Akteur/innen erwartete Verteilung der Erträge ihrerseits einen Einfluss auf die ökonomische Wertschöpfung hat. Beispielseise könnten marktwirtschaftliche Gewinnanreize, die unvermeidlich eine gewisse Ungleichverteilung ökonomischer Güter bedingen, die Produktion wertvoller Ressourcen soweit befördern, dass - ggf. dank steuerlicher Umverteilung - auch die am schlechtesten gestellten Mitglieder der Gesellschaft immer noch in einer sozialen Lage sind, die besser ist als die Lage der am schlechtesten gestellten Mitglieder jeder möglichen Alternativgesellschaft, deren Grundstruktur eine gleichmäßigere Verteilung ökonomischer Ressourcen zur Folge hat. Wie Wirtschaftsordnung und Sozialstaat konkret organisiert werden müssen und wie groß etwa das optimale Ausmaß steuerlicher Umverteilung sein muss, damit das Differenzprinzip erfüllt ist, lässt sich nicht allein aus Rawls' Theorie politischer Gerechtigkeit ableiten. Hierzu bedarf es zusätzlicher empirischsozialwissenschaftlicher Annahmen. Das Differenzprinzip gibt nur ein allgemeines Kriterium vor, an dem sich die empiriegestützte Verteidigung oder Kritik konkreter Regelungsvorschläge orientieren soll. Die vorangehenden Überlegungen bezüglich ökonomischer Ressourcen gelten nicht auch für grundlegende Freiheitsrechte oder gesellschaftliche Positionen. Sie lassen sich beispielsweise nicht in gleicher Weise vermehren wie ökonomische Ressourcen. Daher gibt es auch keinen Grund, bezüglich dieser Freiheitsrechte und Chancen von der Gleichverteilung abzuweichen. Vor allem sind sie für die Integrität, die fairen Lebenschancen und den moralischen Status der Person so grundlegend, dass kein/e Teilnehmer/in des Urzustandes in Kauf nehmen kann, diesbezüglich schlechter gestellt zu sein. Die Parteien des Urzustands werden deshalb auf einer Gleichverteilung beharren.

Gerechtigkeit versus Utilitarismus: Rawls verfolgt mit seiner Theorie der Gerechtigkeit erklärtermaßen das Ziel, eine klar formulierte und systematisch ausgearbeitete santische`Alternative zum Utilitarismus $\mathrm{zu}$ verteidigen, der das angelsächsische Denken lange dominiert habe (ebd., u. a. S. xvii ff., 19 ff.). Denn Rawls, der zunächst selbst zur Weiterentwicklung des Regelutilitarismus beigetragen hatte (Rawls 1955), ist überzeugt, dass der Utilitarismus der wesentlichen Bedeutung individueller Grundrechte nicht gerecht wird (Rawls 1999, S. xii). Sein zentraler Einwand richtet sich gegen den Grundsatz der interindividuellen Nutzenaggregation (siehe Kap. 5.1). Dem Nutzenprinzip des Gesamtnutzenutilitarismus zufolge sind zwei Weltzustände moralisch gleichwertig, wenn die in ihnen jeweils realisierte Nutzensumme, z. B. die Gesamtsumme des Glücksempfindens aller empfindungsfähigen Wesen, gleich groß ist. (Im Fall des Durchschnittsnutzen-Utilitarismus gelten die Weltzustände als gleichwertig, wenn der Quotient aus der Gesamtsumme dividiert durch die Zahl der empfindungsfähigen Wesen gleich groß ist.) Der Utilitaris- 
Kritik des Utilitarismus

Integrität der Person

Rawls 1999, S. 87 mus (in beiden Varianten) behandelt also die Verteilung von Nutzen und Schaden zwischen den Individuen als völlig irrelevant. Aus utilitaristischer Sicht müssen gesellschaftliche Entscheidungen genauso getroffen werden, als ob die individuellen Glücks- oder Leiderfahrungen aller handlungsbetroffenen Wesen diejenigen einer einzigen Person wären, die ihren individuellen Nutzen zu maximieren sucht. Dieses radikal kollektivistische Bild des Verhältnisses zwischen Individuum und Gemeinschaft verstößt Rawls zufolge gegen tief verwurzelte und weithin geteilte Auffassungen. Der Utilitarismus nimmt die Grenzen zwischen Individuen nicht hinreichend ernst (ebd., S.24).

Die natürliche Lotterie: Demgegenüber verteidigt Rawls vehement die Unverletzlichkeit (inviolability, ebd., S. 3, 24, 513) moralischer Personen: die Unveräußerlichkeit ihrer Grundfreiheiten, die Unverrechenbarkeit ihrer subjektiven Erlebnisse, ihre Unvertretbarkeit als Individuen, die über ein eigenes Konzept des guten Lebens verfügen und Anerkennung als eigenständige, mit einem Gerechtigkeitssinn ausgestattete politisch-moralische Akteure verdienen. Diese Integritätsansprüche moralischer Personen beinhalten jedoch kein Anspruchsrecht auf soziale Vorteile, die durch die kontingenten Eigenschaften konkreter Individuen, ihre individuellen Fähigkeiten und Begabungen, ermöglicht werden. Denn zum einen sind auch solche Vorteile in vielfältiger Weise von den Strukturen gesellschaftlicher Kooperation abhängig. Zum anderen stellt die Verteilung individueller Fähigkeiten und Talente in wesentlichem Ausmaß das Ergebnis einer natürlichen Lotterie dar. Es muss daher vom moralischen Standpunkt aus als willkürlich gelten. Selbst das Kriterium der Leistungsgerechtigkeit muss insoweit relativiert werden, da auch die individuelle Leistungsbereitschaft noch von zufälligen Bedingungen wie einem glücklichen Elternhaus und günstigen sozialen Umständen beeinflusst wird (ebd., S. 64). Rawls hält es daher für angemessen, auch die Verteilung der aus natürlichen Talenten und Fähigkeiten resultierenden Vorteile als Frage der politischen Gerechtigkeit zu behandeln und dem Differenzprinzip zu unterwerfen. Auch in Hinsicht auf diese Vorteile zielt das Prinzip nicht darauf, soziale Differenzen, die sich aus natürlichen Unterschieden ergeben, zu eliminieren, sondern ihnen instititutionell so Rechnung zu tragen, dass dies für die am meisten Benachteiligten den (verglichen mit anderen Gesellschaftsordnungen) größten Nutzen bringt. Eben darin besteht der moralisch rationale Umgang mit der Willkür der natürlichen Lotterie, nämlich derjenige Umgang, der von den Parteien hinter dem Schleier des Nichtwissens klugerweise gewählt würde:

"The difference principle represents, in effect, an agreement to regard the distribution of natural talents as in some respects a common asset and to share in the greater social and economic benefits made possible by the complementarities of this distribution. Those who have been favored by nature, whoever they are, may gain from their good fortune only on terms that improve the situation of those who have lost out. The naturally advantaged are not to gain merely because they are more gifted, but only to cover the costs of training and education and for using their endowments in ways that help the less fortunate as well. No one deserves his greater natural capacity nor merits a more favorable starting place in society. But, 
of course, this is no reason to ignore, much less to eliminate these distinctions. Instead, the basic structure can be arranged so that these contingencies work for the good of the least fortunate. Thus we are led to the difference principle if we wish to set up the social system so that no one gains or loses from his arbitrary place in the distribution of natural assets or his initial position in society without giving or receiving compensating advantages in return."

Plausibilität der Gerechtigkeitsprinzipien: Wie oben ausgeführt, hält Rawls das vertragstheoretische Entscheidungsmodell des Urzustands zunächst deshalb für angemessen, weil es (wie die Prozedur des fairen Kuchenteilens) vor-theoretischen Fairnessvorstellungen entspricht. Damit ist jedoch nur der erste Schritt des Begründungswegs zurückgelegt. Insgesamt stellt sich der Rechtfertigungszusammenhang der rawlsschen Ethik wie folgt dar: Wohlerwogene vor-theoretische Annahmen (1) rechtfertigen die Angemessenheit des Entscheidungsmodells »Urzustand « (2); aus der Durchführung des Gedankenexperiments resultieren (nachdem alternative Prinzipienkandidaten erwogen wurden) die beiden Grundsätze der Gerechtigkeit (3). Entsprechend der kohärentistischen Methode des Überlegungsgleichgewichts kann die Rechtfertigung ethischer Prinzipien jedoch nicht nur in eine Richtung verlaufen. Vielmehr sind auch die Prinzipien bzw. ihre paradigmatischen Anwendungen noch einmal daraufhin zu prüfen, inwieweit sie mit unseren wohlerwogenen vor-theoretischen Gerechtigkeitsannahmen vereinbar sind (4). Sollten die aus dem Entscheidungsmodell hervorgehenden Moralprinzipen Konsequenzen zeitigen, die eindeutig gegen fest verwurzelte moralische Intuitionen verstoßen, könnte dies ein Grund für Revisionen der Methode sein. Bezüglich der Methode des Urzustands sei das jedoch nicht der Fall (ebd., S. $17 \mathrm{f}$.). Ihr Ergebnis sei nicht nur konsistent mit unseren wohlerwogenen Gerechtigkeitsannahmen, sie trage auch zu deren Klärung und Systematisierung bei. Der Zusammenhang der verschiedenen Elemente der rawlsschen Gerechtigkeitstheorie lässt sich also in etwa so verstehen, wie in der folgenden Abbildung dargestellt (siehe Abb. 7.1).

Weiterentwicklungen: In späteren Publikationen hat Rawls seine in der Theorie der Gerechtigkeit entwickelte Position in einigen Details revidiert und weiterentwickelt, ohne jedoch ihren Grundgedanken aufzugeben. In seinem Buch Political Liberalism (1993a) versucht er insbesondere, der Tatsache des in demokratischen Gesellschaften zu erwartenden Wertepluralismus deutlicher Rechnung zu tragen. Entsprechend rückt hier das bereits in der Theory of Justice (Rawls 1999, S. 340) herangezogene Konzept des overlapping consensus in den Mittelpunkt. Rawls ist überzeugt, dass sich in den unterschiedlichen weltanschaulichen und religiösen Weltdeutungen und Wertsystemen, die Rawls comprehensive doctrines nennt, ein Grundkonsens auffinden lässt, und dass dieser Grundkonsens insbesondere auch grundlegende Fairnessüberzeugungen umfasst. Darauf soll die Konzeption politischer Gerechtigkeit aufbauen können. Rawls spricht von einem überlappenden Konsens, weil sich übereinstimmende Positionen aus durchaus unterschiedlichen weltanschaulichen Quellen speisen können sollen: Übereinstimmung besteht hinsichtlich moralischer Auffassungen, nicht notwendigerweise auch hin-

Aufbau der Gesamttheorie

Overlapping consensus 
Abb. 7.1: Schema der rawlsschen Gerechtigkeitstheorie
1. Schritt (kohärentistisch)
Wir bestimmen auf Basis vortheoretischer Überzeugungen eine faire Entscheidungsprozedur.

\section{Schritt (kontraktualistisch)}
Im Rahmen dieser (gedanken- experimentellen) Prozedur einigen sich Vertreter/innen sozialer Gruppen hinter dem „veil of ignorance“...

...auf 2 Prinzipien für die Grundstruktur der Gesellschaft...

\section{Vorläufiges Ergebnis}

overlapping consensus

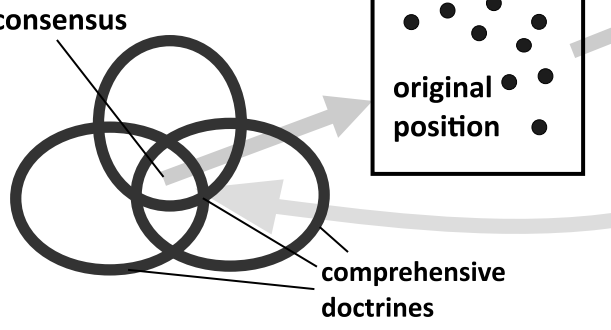

\section{two principles} of justice

4. Schritt (kohärentistisch)

...die als Vorschlag im realen politisch-ethischen Diskurs erneut geprüft werden können.

sichtlich ihrer Begründungen. Entsprechend soll die Konzeption politischer Gerechtigkeit nicht von einzelnen Weltanschauungen abhängen, sondern ihnen gegenüber freistehend (freestanding) sein. Dies soll es möglich machen, einer Gesellschaft, die im Wesentlichen gemäß den in der Theorie der Gerechtigkeit vorgeschlagenen Prinzipien organisiert ist, ein hinreichendes Maß an Stabilität zu sichern. Die dem overlapping consensus im Rahmen der weiterentwickelten Theorie zuerkannte Rolle ist in obiger Grafik bereits angezeigt.

\subsection{4 | Reaktionen und Ausblick}

Die herausragende Bedeutung der rawlsschen Gerechtigkeitstheorie für die Entwicklung der Moralphilosophie im Allgemeinen und die politische Ethik im Besonderen manifestiert sich auch in der Fülle der Reaktionen - konstruktiven Anknüpfungen und Weiterentwicklungen, Kritiken und Gegenentwürfen. Sie beziehen sich sowohl auf die beiden Elemente der von Rawls vorgeschlagenen Begründungsmethode (Überlegungsgleichgewicht und Urzustand) als auch auf die moralische Substanz seiner Gerechtigkeitsethik.

Überlegungsgleichgewicht: Die Methode des Überlegungsgleichgewichts ist in der allgemeinen normativen Ethik und nicht zuletzt auch in den Kontexten der angewandten Ethik vielfach aufgegriffen worden (u. a. Arras 2007; Daniels 1996; van den Burg/van Willigenburg 1998). Sie stellt gegenwärtig die prominenteste Methode ethischer Rechtfertigung dar (Tersman 2018, S. 1). Nicht wenigen gilt sie überhaupt als einziger ernst zu nehmender Kandidat einer möglichen ethischen Begründungsmethode (Scanlon 2002; vgl. Kelly/McGrath 2010). Sie ist aber auch Gegenstand mehr oder weniger radikaler Kritik (de Maagt 2017).

Zunächst sieht sie sich Einwänden ausgesetzt, die gegen den Kohärentismus generell vorgebracht werden. Eine Kritik setzt an der Tatsache an, 
dass den wohlerwogenen Gerechtigkeitsurteilen oder Intuitionen, die den Ausgangspunkt der auf ein Überlegungsgleichgewicht zielenden Rechtfertigungsbemühung bilden, eine gewisse Anfangsplausibilität zugeschrieben werden muss, wenn das Verfahren zu irgendeiner wertvollen Erkenntnis führen soll. Ist diese Zuschreibung berechtigt? Die Methode sieht sich an dieser Stelle ähnlichen Zweifeln ausgesetzt wie der Intuitionismus (siehe Kap. 8.2). Dagegen wird angeführt, dass schlechthin voraussetzungslose Begründungen unmöglich und ein ethischer Skeptizismus, der die Haltbarkeit aller moralischen Überzeugungen bezweifelt, äußerst unplausibel sei.

Häufig vorgebracht wird der Vorwurf der Vagheit (zumindest der klassischen Formulierungen) der Methode des Überlegungsgleichgewichts (Hahn 2000). Einen Ansatzpunkt dafür liefert der für die Methode charakteristische Umstand, dass im Fall einer Inkonsistenz oder Spannung zwischen mehreren theoretischen oder vor-theoretischen Überzeugungselementen Konsistenz grundsätzlich auf mehrere Arten hergestellt werden kann. Inwieweit diese Eigenschaft einen problematischen Relativismus zur Folge hat, wird kontrovers diskutiert (Agostino 1988; Nielsen 1993). Unbestimmt ist die Methode jedenfalls auch insofern, als sie mit unterschiedlichen metaethischen Positionen vereinbar scheint (Darwall/ Gibbard/Railton 1992, S.123). Dies kann man freilich auch als Vorteil sehen; die Popularität der Methode hat es zweifellos begünstigt (vgl. exemplarisch Brink 1989, Audi 1998, Scanlon 2014). Diskutiert wird auch, inwieweit die Methode selbsttragend ist oder vielmehr normative Prinzipien bereits voraussetzt (insbes. logische Normen oder eine allgemeine Kohärenzverpflichtung) und inwieweit ein Begründungsverfahren, das auf die Herstellung von Kohärenz im Rahmen eines faktisch bereits existierenden Überzeugungssystems zielt, überhaupt der Aufgabe der Begründung normativer Gehalte gerecht werden kann. Läuft dieser Versuch nicht auf einen Sein-Sollens-Fehlschluss (siehe Kap.4.1) hinaus? Darauf lässt sich erwidern, dass die Ausgangsüberzeugungen selbst bereits normativer Natur sind, also nicht von einem neutralen Sein auf ein Sollen geschlossen wird (Hahn 2016).

Urzustand: Im Hinblick auf das Entscheidungsmodell des Urzustands ist behauptet worden, dass die Parteien nicht zwangsläufig zu dem behaupteten Ergebnis kommen, sich also nicht unbedingt auf die von Rawls vorgeschlagenen Gerechtigkeitsprinzipien einigen würden (Sen 2009, S. 11 f., 56 ff., 90). Aus Sicht des Kommunitarismus (s. u.) ist zudem eingewandt worden, dass den Parteien hinter dem Schleier des Nichtwissens überhaupt zu wenig Informationen zur Verfügung stünden, als dass diese überhaupt noch rationale Entscheidungen treffen könnten. Die Konstruktion des Urzustands bringe einen extremen Individualismus zum Ausdruck: Sie setze die unplausible Annahme voraus, dass wir auch dann noch rationale Entscheidungsgründe hätten, wenn wir von unseren durch unseren konkreten sozialen Lebenskontext geprägten Vorstellungen des guten Lebens radikal abstrahieren. Zwar sei die wechselseitige Gleichgültigkeit der Parteien im Urzustand nicht als Merkmal einer Idealgesellschaft gedacht, sondern nur als Element des Gedankenexperiments, das die Idee Unparteilichkeit modellieren soll. Die Annahme der wechsel-

Kommunitaristische Kritik 
seitigen Gleichgültigkeit der hypothetischen Vertragsparteien sei gleichwohl problematisch, weil er den Parteien den Zugang zu den Quellen vernünftiger Wertentscheidungen abschneide (Sandel 1982; für eine klare Rekonstruktion vgl. Kelly 1994). Dem wird entgegengehalten, dass für Entscheidungen über die Verteilung der relativ abstrakten Freiheiten und Güter, die im Urzustand zur Debatte stehen, spezifische Informationen über die Wertsysteme der Betroffenen nicht nötig seien (Freeman 2016). In jedem Fall liegt im Modell des Urzustands eine gewisse Ambivalenz: Einerseits soll Rawls' Kontraktualismus die Idee der Unparteilichkeit auf eine Weise ausdrücken, die der moralischen Bedeutung der Grenzen zwischen Personen Rechnung trägt. Andererseits gehen die Abstraktionen des Urzustands so weit, dass den hypothetischen Vertragsparteien ihre wechselseitigen Unterschiede verborgen bleiben. Einerseits adressiert Rawls' politische Ethik erklärtermaßen Personen, die bereits über einen Gerechtigkeitssinn verfügen. Andererseits sollen diese Personen sich hypothetisch in die Rolle rational-egoistischer Vorteilsmaximierer versetzen, wobei sie aber gleichwohl noch Zugang zu den wesentlichen Gründen für eine Entscheidung zwischen alternativen Gerechtigkeitsprinzipien haben sollen (Habermas 1995).

Begründungsanspruch: Unklar schien zunächst auch, ob der Urzustand Teil einer philosophischen Gerechtigkeitskonzeption ist, die sich gegenüber allen Personen unabhängig von deren faktischen Überzeugungen als verbindlich ausweisen lässt, oder vielmehr Element einer politischen Konzeption der Gerechtigkeit, die als Vorschlag zur gesellschaftlichen Selbstverständigung zu verstehen ist. Rawls legt sich in den 1980er Jahren auf die zweite Lesart fest. Der Urzustand diene nur der anschaulichen »Repräsentation" des Kernbereichs eines überlappenden Konsenses (kernel of an overlapping consensus, Rawls 1985, S. 246) über Fragen der politischen Gerechtigkeit. Diese Interpretation bedeutet zum einen eine Abschwächung des durch Rawls' Theorie erhobenen Begründungsanspruchs. Zum anderen beinhaltet sie das Zugeständnis, dass alternative Modelle den Kernbereich unserer wohlerwogenen Fairnessüberzeugungen grundsätzlich ebenso angemessen repräsentieren könnten. Als denkbare Konkurrenzmodelle kämen etwa das Konzept des unparteilichen Beobachters (Kymlicka 1993; siehe Kap.4) oder das idealisierende Modell des argumentativen Diskurses (Habermas 1995) in Frage.

Maximin-Prinzip: Kritik richtet sich auch gegen Rawls' Annahme, es

Kritik an Maximinund Differenzprinzip sei für die Parteien im Urzustand rational, sich am Maximin-Prinzip zu orientieren. Könnten sie eine risikofreudigere Strategie wählen und entsprechend für eine Einrichtung der Grundstruktur optieren, die zwar nicht die Lebensaussichten der am schlechtesten gestellten Mitglieder der Gesellschaft optimiert, aber ihnen für den Fall, dass sie sich in doch in einer günstigeren sozialen Situation wiederfinden, bessere Aussichten verspricht? Rawls versucht von der Wahl solcher Strategien dadurch abzuschrecken, dass er vollkommene Ungewissheit auch über die Wahrscheinlichkeiten postuliert, mit der Parteien eine bestimmte soziale Position innehaben. Das Maximin-Prinzip sei insofern rational, als es die Parteien davon abhalte, einer Grundstruktur zuzustimmen, die für die am schlechtesten gestellten Personen unerträgliche Lebensbedingungen be- 
deuten würde (Rawls 2001, S. 98 ff.). Der Streit um die Frage, ob im Urzustand das Maximin-Prinzip akzeptiert würde, verliert allerdings durch die Deutung der rawlsschen Gerechtigkeitskonzeption als politische Konzeption an Brisanz. Denn wie der Urzustand überhaupt wird durch dieses Zugeständnis auch das Maximin-Prinzip auf ein bloß heuristisches Prinzip (simply a useful heuristic device, ebd., S. 99) herabgestuft: Es muss als Teil von Rawls' Deutungsangebot für den Kernbereich unserer Fairnessüberzeugungen verstanden werden, nicht als Teil einer unabnhängigen Begründungsbasis für dieses Deutungsangebot.

Differenzprinzip: Von unveränderter Bedeutung bleibt damit jedoch die gleichfalls schon früh diskutierte Frage, ob das eng mit dem MaximinPrinzip verbundene Differenzprinzip für die Verteilung sozialer Ressourcen insgesamt plausibel ist. Gehört dieses Prinzip tatsächlich von dem von Rawls postulierten Kernbereich geteilter Fairnessüberzeugungen oder folgt es jedenfalls aus ihnen? Kritiker/innen halten den Fokus auf die Optimierung der Situation der am meisten Benachteiligten für überzogen, für zu starr (Koller 1983) oder überhaupt für unplausibel (Nozick 1974, S. 189 ff.). Befürworter/innen des Differenzprinzips rechtfertigen es unter anderem mit dem Hinweis auf Vorstellungen demokratischer Gleichheit (Cohen 1989). Die von beiden Seiten angeführten Argumente machen in vielfältiger Weise von politisch-ethischen Intuitionen und Konzepten und teils auch empirischen Annahmen Gebrauch. Sie entziehen sich insofern Versuchen einer knappen Systematisierung.

Capabilities Approach: Aus der Fülle der durch Rawls angeregten Diskussionen und Alternativentwürfe müssen drei besonders sichtbare Strömungen zumindest erwähnt werden: Der Fähigkeiten-Ansatz, der Libertarismus und der Kommunitarismus. Der Fähigkeiten-Ansatz (capabilities approach) teilt viele der Grundannahmen und politisch-ethischen Überzeugungen, die in Rawls' Gerechtigkeitstheorie repräsentiert sind (Sen 1979; Nussbaum/Sen 1993; Nussbaum 2011). Als primärer Gegenstand von Gerechtigkeitsansprüchen müssen ihm zufolge jedoch nicht soziale Güter, sondern grundlegende Fähigkeiten (capabilities) der Anspruchssubjekte gelten. Diese These verdankt sich zum einen der (u. a. in entwicklungspolitischen Kontexten gewonnenen) Einsicht, dass der konkrete Nutzen von Gütern für die Aufrechterhaltung und Entwicklung menschlicher Fähigkeiten in hohem Maße von den Lebensbedingungen der über die Güter verfügenden Individuen abhängt (Sen 1993). Zum anderen speist sie sich aus einem Konzept menschlicher Selbstentfaltung, das von einem Spektrum allgemein menschlicher Grundfunktionen ausgeht. Vor allem in Nussbaums früheren Schriften war dieses Konzept stark von Aristoteles' naturalistischer Tugendethik (siehe Kap. 2.2) inspiriert, in ihren späteren Arbeiten wird es verstärkt auch auf Vorstellungen von Menschenwürde bezogen (Nussbaum 2011, S. 123 ff.). Daraus erhellt, dass der Fähigkeiten-Ansatz als solcher nicht notwendigerweise an einen bestimmten moralphilosophischen Grundansatz gebunden ist. So bemühen sich Claassen und Düwell um eine an Alan Gewirth statt Aristoteles orientierte Grundlegung des Fähigkeiten-Ansatzes (Claassen/ Düwell 2013).

Libertarismus und Kommunitarismus: In den an Rawls anschließen- 
Rawls' normativer Individualismus den politisch-ethischen Diskussionen sind schon früh zwei Strömungen namhaft gemacht worden, die als Libertarismus und Kommunitarismus bekannt sind (vgl. knapp Bell 2016; Van der Vossen 2019; ausführlich Reese-Schäfer 1997; als Anthologie Honneth 1992). Es handelt sich dabei nicht nur um akademisch-wissenschaftliche, sondern auch um ideologisch-politische Bewegungen: Libertaristen haben in den USA eigene politische Partei gegründet und sind auch in der Republikanischen Partei stark repräsentiert. Kommunitaristische Ideen sind wiederum sowohl von gemäßigt sozialdemokratischer Seite, etwa von Tony Blairs "New Labour ", als auch von gesellschaftspolitisch konservativen Strömungen aufgegriffen worden. Als philosophische Schulen setzen sich Libertarismus und Kommunitarismus in gegenläufige Richtungen von Rawls' politischer Ethik ab. Die Absetzungsbewegungen lassen sich grob charakterisieren, wenn man sie als Reaktion auf die von Rawls vertretene spezifische Form des normativen Individualismus interpretiert: Begreift man Individualismus als eine Position, welche die Ansprüche von Individuen als grundlegend und/oder vorrangig betrachtet und Kollektivismus als eine, die den Ansprüchen von Gruppen oder Gemeinschaften diese Rolle zuweist, so vertritt Rawls nachdrücklich einen normativen Individualismus. Denn die Quelle normativer Ansprüche sieht er im moralischen Status und den Ansprüchen individueller Personen. Diese Ansprüche müssen allerdings unparteiisch geprüft werden, wobei faktische Ungleichheiten (im Hinblick auf Macht, Eigentum, Fähigkeiten etc.) grundsätzlich auf ihre Legitimität hin zu überprüfen sind. Vertreter/innen des Kommunitarismus wenden sich nun gegen den normativen Individualismus der rawlsschen Gerechtigkeitstheorie sowie die damit verbundene Behauptung eines Vorrangs des Gerechten vor dem Guten. Vertreter/innen des Libertarismus geht der rawlssche Individualismus hingegen gerade nicht weit genug. Sie vertreten eine starke, an John Locke anknüpfende Eigentumstheorie. Diese Theorie assimiliert die Rechte an der eigenen Person an Eigentumsrechte und Eigentumsrechte wiederum an vor-politische Rechte auf Schutz der personalen Integrität. Entsprechend dieser Auffassung betrachten sie jede Besteuerung oder sonstige Umverteilung rechtmäßig erworbenen Eigentums als eine Verletzung von Persönlichkeitsrechten und setzen sie gar mit Zwangsarbeit gleich (Nozick 1974, S. 169). Die vom Liberatismus in Anspruch genommene Eigentumstheorie ist allerdings grundlegenden Einwänden ausgesetzt (vgl. knapp Werner 2012).

Ausblick: John Rawls' deontologische Ethik politischer Gerechtigkeit misst die Gerechtigkeit gesellschaftlicher Basisinstitutionen an zwei Grundsätzen. Der erste Grundsatz spricht allen Bürger/innen dieselben Grundfreiheiten zu. Der zweite Grundsatz legt fest, dass Ungleichheiten in der Verteilung sozialer Ressourcen nur unter zwei Bedingungen legitim sind. Erstens müssen die Ungleichheiten aus einer fairen und inklusiven sozialen Kooperation und Konkurrenz hervorgehen. Zweitens muss die resultierende Verteilung für die am schlechtesten gestellten Mitglieder der Gesellschaft günstiger sein als es die Verteilung, die sich aus der Kooperation in jeder alternativen Gesellschaftsordnung ergeben würde, für die dort jeweils am meisten benachteiligte Gruppe wäre. Rawls möchte zeigen, dass die beiden Gerechtigkeitsgrundsätze aus Fairnessüberzeugun- 
gen herzuleiten sind, die einen Teil unseres weltanschauungsübergreifenden Grundkonsenses (overlapping consensus) ausmachen. Dies sucht er durch das vertragstheoretische Gedankenexperiment des Urzustands plausibel zu machen, das unsere Fairnessüberzeugungen auf transparente Weise nachmodellieren soll.

Wer sich mit sozialer Gerechtigkeit oder mit der Ethik politischer Institutionen beschäftigt, kommt an der Auseinandersetzung mit Rawls nicht vorbei. Seine Kritik des Utilitarismus und sein sorgfältig ausgearbeitetes Modell einer von Kant beeinflussten politischen Gerechtigkeitsethik haben die Diskussion stark geprägt. Rawls' Einfluss auf die Diskussion über ethische Begründungsmethoden ist nicht minder bedeutsam. Sein Konzept des Überlegungsgleichgewichts hat wesentlich dazu beigetragen, die Moralphilosophie nach einer Zeit metaethisch begründeter Skepsis (siehe Kap.8) als seriöse und aussichtsreiche Disziplin zu rehabilitieren. Es wird weit über den Bereich der politischen Ethik hinaus in Anspruch genommen und weiterentwickelt - in der allgemeinen Moralphilosophie ebenso wie in den verschiedenen Feldern bereichsspezifischer Ethik (siehe Kap. 11).

\section{Siglenverzeichnis}

AA - Akademieausgabe (Kant 1902 ff.)

KSA - Kritische Studienausgabe (Nietzsche 1988)

SEP - Stanford Encyclopedia of Philosophy (Zalta)

\section{Einführende Literatur}

Freeman, Samuel: The Cambridge Companion to Rawls. Cambridge/New York 2003.

Pogge, Thomas W.: John Rawls: His Life and Theory of Justice. Oxford/New York 2007.

Rawls, John: Justice as Fairness: A Restatement. Cambridge/London 2001.

\section{Zitierte und weiterführende Literatur}

Agostino, F. D.: »Relativism and Reflective Equilibrium in Justification«. In: The Monist 71/3 (1988), S. 420-436.

Apel, Karl-Otto: Transformation der Philosophie. Frankfurt a. M. 1973, Bd. 2, S. 358-435.

Apel, Karl-Otto: Diskurs und Verantwortung. Frankfurt a. M. 1988.

Audi, Robert: "Moderate Intuitionism and The Epistemology of Moral Judgment«. In: Ethical Theory and Moral Practice 1 (1998), S. 15-44.

Bell, Daniel: »Communitarianism«. In: SEP 2016.

Boucher, David/Kelly, Paul J. (Hrsg.): The Social Contract from Hobbes to Rawls. London/New York 1994.

Buber, Martin: Ich und Du [1923]. Stuttgart 1995.

Brink, David O.: Moral Realism and the Foundation of Ethics. Cambridge/New York 1989.

Brune, Jens P.: Moral und Recht: Zur Diskurstheorie des Rechts und Demokratie von Jürgen Habermas. Freiburg i. Br. 2010.

Claassen, Rutger/Düwell, Marcus: »The Foundations of Capability Theory: Comparing Nussbaum and Gewirth". In: Ethical Theory and Moral Practice 16/3 (2013), S. 493-510.

Cohen, Joshua: »Democratic Equality«. In: Ethics 99/4 (1989), S. 727-751.

Daniels, Norman: Justice and Justifcation: Reflective Equilibrium in Theory and Practice. Cambridge/New York 1996. 
Darwall, Stephen L.: The Second-Person Standpoint: Morality, Respect, and Accountability. Cambridge/London 2006.

Darwall, Stephen L./Gibbard, Allan/Railton, Peter: »Toward Fin de siècle Ethics: Some Trends«. In: The Philosophical Review 101/1 (1992), S. 115-189.

de Maagt, Sem: »Reflective Equilibrium and Moral Objectivity«. In: Inquiry 60/5 (2017), S. 443-465.

Fichte, Johann G.: Gesamtausgabe der Bayerischen Akademie der Wissenschaften. Stuttgart-Bad Cannstatt $1962 \mathrm{ff}$.

Forst, Rainer: Das Recht auf Rechtfertigung: Elemente einer konstruktivistischen Theorie der Gerechtigkeit. Frankfurt a. M. 2005.

Freeman, Samuel: »Original position«. In: SEP 2016.

Gadamer, Hans-Georg: Wahrheit und Methode: Grundzüge einer philosophischen Hermeneutik. In: Gesammelte Werke, Bd. 1. Tübingen ${ }^{7} 2010$.

Gert, Bernard: Morality: Its Nature and Justification. New York/Oxford 1998.

Gilligan, Carol: In a Different Voice. Cambridge 1982.

Habbel, Torsten: Der Dritte stört: Emmanuel Levinas, Herausforderung für politische Theologie und Befreiungsphilosophie. Mainz 1994.

Habermas, Jürgen: Theorie des kommunikativen Handelns. 2 Bände. Frankfurt a. M. 1981 .

Habermas, Jürgen: Nachmetaphysisches Denken: Philosophische Aufsätze. Frankfurt a. M. 1988

Habermas, Jürgen: »Reconciliation through the Public Use of Reason: Remarks on John Rawls's Political Liberalism«. In: The Journal of Philosophy 92/3 (1995), S. 109-131.

Hahn, Susanne: Überlegungsgleichgewicht(e): Prüfung einer Rechtfertigungsmetapher. Freiburg i. Br. 2000.

Hahn, Susanne: »From Worked-out Practice to Justified Norms by Producing a Reflective Equilibrium«. In: Analyse \& Kritik 38/2 (2016), S. 333-369.

Hegel, Georg W.: Werke in zwanzig Bänden. Frankfurt a. M. 1970.

Held, Virginia: The Ethics of Care: Personal, Political, and Global. Oxford/New York 2006.

Honneth, Axel (Hrsg.): Kommunitarismus: Eine Debatte über die moralischen Grundlagen moderner Gesellschaften. Frankfurt a. M./New York 1992.

Honneth, Axel: Anerkennung: Eine europäische Ideengeschichte. Berlin 2018.

Kant, Immanuel: Gesammelte Schriften, herausgegeben von der Königlich Preußischen Akademie der Wissenschaften. Berlin $1902 \mathrm{ff}$.

Kelly, Paul J.: »Justifying `Justices: Contractarianism, Communitarianism and the Foundations of Contemporary Liberalism«. In: Boucher, David/Kelly, Paul J. (Hrsg.): The Social Contract from Hobbes to Rawls. London/New York 1994, S. 227-245.

Kierkegaard, Søren: Entweder - Oder [1843]. München 2000.

Koller, Peter: »Rawls’ Differenzprinzip und seine Deutungen«. In: Erkenntnis 20/1 (1983), S. 1-25.

Kymlicka, Will: »The Social Contract Tradition«. In: Singer, Peter (Hrsg.): A Companion to Ethics. Oxford 1993, S. 186-196.

Levinas, Emmanuel: Totalität und Unendlichkeit: Versuch über die Exteriorität. Freiburg i. Br./München Alber 1987.

Levinas, Emmanuel: Humanismus des anderen Menschen. Hamburg 1989.

Lukács, Georg: Geschichte und Klassenbewußtsein. Neuwied/Berlin 1970.

Lukács, Georg: Die Zerstörung der Vernunft. Berlin 1955.

Nielsen, Kai: »Relativism and Wide Reflective Equilibrium«. In: The Monist 76/3 (1993), 316-332.

Nietzsche, Friedrich: Sämtliche Werke: Kritische Studienausgabe in 15 Einzelbänden. München/Berlin/New York ${ }^{2} 1988$.

Nozick, Robert: Anarchy, State, and Utopia. New York 1974.

Nussbaum, Martha C./Sen, Amartya (Hrsg.): The Quality of Life. Oxford 1993. 
Nussbaum, Martha C.: Creating Capabilities. The Human Development Approach. Cambridge/London 2011.

Olsson, Erik: »Coherentist Theories of Epistemic Justification«. In: SEP 2017.

Popper, Karl R.: Gesammelte Werke in deutscher Sprache. Tübingen ${ }^{7} 2003$.

Rawls, John: »Outline of a Decision Procedure for Ethics«. In: The Philosophical Review 60/2 (1951), S. 177-197.

Rawls, John: »Two Concepts of Rules«. In: The Philosophical Review 64/1 (1955), S. 3-32.

Rawls, John: Eine Theorie der Gerechtigkeit. Frankfurt a. M. 1975.

Rawls, John: »Justice as Fairness: Political not Metaphysical«. In: Philosophy and Public Affairs 14/3 (1985), S. 223-251.

Rawls, John: A Theory of Justice. Revised Edition. Cambridge 1999.

Rawls, John: Political Liberalism. New York 1993a.

Rawls, John: »The Law of Peoples«. In: Critical Inquiry 20/1 (1993b), S. 36-68.

Rawls, John: Politischer Liberalismus. Frankfurt a. M. 1998.

Rawls, John: Justice as Fairness: A Restatement. Cambridge/London 2001.

Rawls, John: Gerechtigkeit als Fairneß: Ein Neuentwurf. Frankfurt a. M. 2003.

Reese-Schäfer, Walter: Grenzgötter der Moral: Der neuere europäisch-amerikanische Diskurs zur politischen Ethik. Frankfurt a. M. 1997.

Riedel, Manfred (Hrsg.): Rehabilitierung der praktischen Philosophie. Freiburg i. Br. 1972.

Rorty, Richard (Hrsg.): The Linguistic Turn: Essays in Philosophical Method. Chicago 1967.

Sandbothe, Mike: Die Renaissance des Pragmatismus. Weilerswist 2000.

Sandkühler, Hans J./De la Vega, Rafael: Marxismus und Ethik: Texte zum neukantianischen Sozialismus. Frankfurt a. M. 1970.

Sandel, Michael: Liberalism and the Limits of Justice. Cambridge 1982.

Scanlon, Thomas M.: »Rawls on Justification«. In: Freeman, Samuel R. (Hrsg.): The Cambridge Companion to Rawls. Cambridge/New York 2002, S. 139-167.

Scanlon, Thomas M.: Being Realistic About Reasons. Oxford/New York 2014.

Scholtz, G.: »Historismus/Historizismus«. In: Ritter, Joachim/Gründer, Karlfried/ Gabriel, Gottfried: Historisches Wörterbuch der Philosophie. Basel/Stuttgart 1971 ff., Bd. 3, Sp. 1141-1147.

Schopenhauer, Arthur: Sämtliche Werke, herausgegeben von Arthur Hübscher. Mannheim ${ }^{4} 1988$.

Schweitzer, Albert: Kultur und Ethik. München 1996.

Sen, Amartya: »Equality of What?«. The Tanner Lecture on Human Values (1979). In: http://www.ophi.org.uk/wp-content/uploads/Sen-1979_Equality-of-What. pdf (20.7.2020).

Sen, Amartya: »Capability and Well-Being«. In: Nussbaum, Martha/Sen, Amartya (Hrsg.): The Quality of Life. Oxford 1993, S. 30-53.

Sen, Amartya: The Idea of Justice. Cambridge 2009.

Siep, Ludwig/Ikaheimo, Heikki/Quante Michael (Hrsg.): Handbuch Anerkennung. Wiesbaden 2018.

Slote, Michael A.: The Ethics of Care and Empathy. New York/London 2007.

Tersman, Folke: »Recent Work on Reflective Equilibrium and Method in Ethics«. In: Philosophy Compass 13/6 (2018), S. e12493.

Theunissen, Michael: Der Andere: Studien zur Sozialontologie der Gegenwart. Berlin 1965.

Van der Burg, Wibren/Van Willigenburg, Theo (Hrsg.): Reflective Equilibrium. Dordrecht 1998.

Van der Vossen, Bas: »Libertarianism«. In: SEP 2019.

Welzel, Hans: Naturrecht und materiale Gerechtigkeit. Göttingen ${ }^{5} 1990$.

Williams, Bernard: Ethics and the Limits of Philosophy [1985]. London/New York 2006. 
Werner, Micha H.: »Property Rights«. In: Chadwick, Ruth F. (Ed.): Encyclopedia of Applied Ethics. San Diego ${ }^{2} 2012$, S. 624-631.

Werner, Micha H.: »Die Unmittelbarkeit der Begegnung und die Gefahr der Dichotomie: Buber, Levinas und Jonas über Verantwortung«. In: Henkel, Anna et al. (Hrsg.): Dimensionen der Sorge. Baden-Baden 2016, S. 99-133.

Zalta, Edward N. (Hrsg.): The Stanford Encyclopedia of Philosophy (SEP). In: https://plato.stanford.edu/.

Open Access Dieses Kapitel wird unter der Creative Commons Namensnennung 4.0 International Lizenz (http://creativecommons.org/licenses/by/4.0/deed.de) veröffentlicht, welche die Nutzung, Vervielfältigung, Bearbeitung, Verbreitung und Wiedergabe in jeglichem Medium und Format erlaubt, sofern Sie den/die ursprünglichen Autor(en) und die Quelle ordnungsgemäß nennen, einen Link zur Creative Commons Lizenz beifügen und angeben, ob Änderungen vorgenommen wurden.

Die in diesem Kapitel enthaltenen Bilder und sonstiges Drittmaterial unterliegen ebenfalls der genannten Creative Commons Lizenz, sofern sich aus der Abbildungslegende nichts anderes ergibt. Sofern das betreffende Material nicht unter der genannten Creative Commons Lizenz steht und die betreffende Handlung nicht nach gesetzlichen Vorschriften erlaubt ist, ist für die oben aufgeführten Weiterverwendungen des Materials die Einwilligung des jeweiligen Rechteinhabers einzuholen. 